\title{
Interactive comment on "Recurrence intervals for the closure of the Dutch Maeslant surge barrier" by Henk W. van den Brink and Sacha de Goederen
}

\section{Anonymous Referee \#2}

\section{Received and published: 31 May 2017}

The paper addresses the assessment of risks associated with high water extreme events reoccurring within a certain period of time with the practical application to the potential surge barrier closures of the Rotterdam Maeslant barrier. The extreme event statistics is constructed based on the seasonal ECMWF forecast data, which provides a set of physically plausible situations and can be considered as a quasi-observational dataset of several thousands years. Additionally, river discharge is approximated for Rotterdam from the 100 years of observations. Extreme value distribution is fitted based on annual maxima of the water level data to estimate the frequency of occurrence. The idea of estimating events inter-arrival times by Poisson process is proposed and rejected due to violation of the independency requirement. Instead, the empirical probability distribution function for lower thresholds is constructed from the dataset and then extrapolated to higher thresholds. It is also shown how linear consideration of sea 
level rise would influence the double closure frequencies within the established model frame.

OSD

The paper is well structured; the goals and results are clearly described. The discussed problematic is relevant and is of both methodological and resultant interest. However, the methodology description and its applications are not always straightforward. In particular, the part concerning Poisson process (section 3.2 and further on) is misleading because, as far as I understood, it is used only to show the falseness of the independency assumption. Presumably, the trial of this method was part of the research process and search for the proper estimate of the reoccurring events. In this case the description of the method seems to be superfluous for this paper.

The usage of forecast members is a valid and effective approach to expand the dataset in the lack of instrumental measurements. Just for understanding, did the authors have 2 model simulations - with and without meteo-forcing, or astronomical tides were analytically estimated (p3. lines 32-33)? Are surge heights used somewhere in this study? It looks like all the data analysis is done based on water level timeseries, in this case, please remove 'surge' from the text (e.g. p3. line 18).

There are several sources of uncertainty emerging throughout the analysis; it would be helpful to see the estimate of total uncertainty range. This is partly done in Figure 8 and section 5.1, but what about the uncertainties from GEV estimate and correction? In the conclusion (lines 40-42) very precise numbers are given without any potential error intervals, an additional sentence or two and a rough estimate in percentage of the total results would suffice here.

Minor comments:

- in abstract, p8 line 9 and maybe somewhere else in the text: reduplicates is presumably used in the meaning of "a factor of two" but cannot be used in this sense and is misleading in the context. Please use other synonyms (doubles, redoubles, duplicates,...)

Interactive comment 
- Eq.5 and others use log and p.3 line80 use In. If log is natural logarithm, please use the same notations everywhere.

OSD

- p5. lines 13: L_adj is actually adjusted surge or rather adjusted water level?

- p3. line 77: please coordinate singular/plural forms The distribution(s) ... are (is)...

- fig. 1 and 4.1: is the correction made by adding these values to the observed data for

Interactive

comment each year? Where the numbers are coming from?

- p5. line 33: how does the value $0.57 \mathrm{~m}$ came from the eq. (1) using $12280 \mathrm{~m}^{\wedge} 3 / \mathrm{s}$ discharge I come out with $0.43 \mathrm{~m}$...

- p5. line 38-39: it is not that the water levels at Rotterdam are $3 \mathrm{~cm}$ higher than at Hoek van Holland, it is that for Rotterdam you consider additionally the river discharge and for $\mathrm{HvH}$ only the sea level. Please reformulate the sentence, it is misleading.

- p5. line 27-28: 'River run-off is not considered in this paper'. Do the authors mean it is not considered in the hydrodynamic model? Because later in the paper there is a talk about run-off again.

- fig.6: upper panel - 2 times "observation" In the legend.

Interactive comment on Ocean Sci. Discuss., doi:10.5194/os-2017-5, 2017. 